\title{
Screened-interaction expansion for the Hubbard model and determination of the quantum Monte Carlo Fermi surface
}

\author{
Gökhan Esirgentit and Heinz-Bernd Schüttler \\ Center for Simulational Physics, Department of Physics and Astronomy, University of Georgia, Athens, Georgia 30602-2451 \\ Carsten Gröber and Hans Gerd Evertz目 \\ Institut für Theoretische Physik, Am Hubland, Universität Würzburg, D-97074 Würzburg, Germany
}

(October 29, 2018)

\begin{abstract}
We develop a systematic self-consistent perturbative expansion for the self energy of Hubbard-like models. The interaction lines in the Feynman diagrams are dynamically screened by the charge fluctuations in the system. Although the formal expansion is exact-assuming that the model under the study is perturbative - only if diagrams to all orders are included, it is shown that for large-on-site-Coulomb-repulsion- $U$ systems weak-coupling expansions to a few orders may already converge. In order to test the approximation at intermediate-to-high temperatures, we use the exact charge-fluctuation susceptibility from quantum Monte Carlo (QMC) simulation studies as input, which determines the exact screened interaction, and compare our results for the self energy to the QMC results. We also make comparisons with fluctuation-exchange (FLEX) approximation. We show that the screened interaction for the large- $U$ system can be vanishingly small at a certain intermediate electron filling; and it is found that our approximation for the imaginary part of the one-particle self energy agrees well with the QMC results in the low energy scales at this particular filling. But, the usefulness of the approximation is hindered by the fact that it has the incorrect filling dependence when the filling deviates from this value. We also calculate the exact QMC Fermi surfaces for the two-dimensional (2-D) Hubbard model for several fillings. Our results near half filling show extreme violation of the concepts of the band theory; in fact, instead of growing, Fermi surface vanishes when doped toward the half-filled Mott-Hubbard insulator. Sufficiently away from half filling, noninteracting-like Fermi surfaces are recovered. These results combined with the Luttinger theorem might show that diagrammatic expansions for the nearly-half-filled Hubbard model are unlikely to be possible; however, the nonperturbative part of the solution seems to be less important as the filling gradually moves away from one half. Results for the 2-D one-band Hubbard model for several hole dopings are presented. Implications of this study for the high-temperature superconductors are also discussed.
\end{abstract}

PACS numbers: 71.10.Fd, 71.18.+y, 71.10.Hf, 74.72.-h

\section{INTRODUCTION}

The basic Hamiltonian for the simplest description of interacting electrons in a periodic potential of fixed lattice ions, the one-band Hubbard model, was widely investigated after the discovery of high-temperature superconductors. In two dimensions, this model is widely accepted to have an antiferromagnetic ground state at half filling of its tight-binding band, although the temperature evolution and the relation with the antiferromagnetism of its insulating electron spectrum is poorly understood. 1 But, there is still ongoing debate about the nature of this model, in fact, there are various mysteries, at band fillings close but not equal to one half. The model may have a superconducting ground state or be close to such an instability at these fillings; therefore, understanding the Hubbard model seems to be crucial for an understanding of the high-temperature superconductors.

There have been numerous approaches to the solution of the two-dimensional (2-D) Hubbard model. Exact diagonalization studies are limited to very small lattices and mainly for this reason they are inconclusive $\mathbf{B}$ Because of statistical errors, exact quantum Monte Carlo (QMC) studies at band fillings corresponding to that of the high-temperature superconductors (about 15\% doped away from one half) are limited to temperatures no less than about a quarter of the electron-hopping energy of the model.t At such moderately high temperatures little information can be obtained about possible lowtemperature instabilities such as superconductivity. But it should be reminded that QMC studies have led to a fairly good understanding of the insulating antiferromagnetic behavior of the half-filled model which corresponds to the undoped parent compounds of the hightemperature superconductors.

To overcome the lattice size or temperature limitations of the available exact methods, approximate methods are needed. An important class of such approximations use diagrammatic formalisms. Since these approximations are weak coupling in nature, it is important that the approximation sums up the physically important diagrams that constitute the exact infinite perturbative expansion. For example, self-consistent approximations like 
fluctuation-exchange (FLEX) approximation, generalizes the physically important Hartree-Fock approximation by adding electron-hole and electron-electron pair scattering events to the bare Coulomb interaction.

In order to go beyond Hartree-Fock- or FLEX-type approximations, one has to employ additional diagrams. A nice formal approach to this is to renormalize, i.e., replace with a perturbative expansion, the bare-interaction lines in the diagrams. But, this can cause an enormous numerical-calculational challenge, because now, the interaction lines, in principle, can depend on the momentum and frequency of the incoming and outgoing electrons, whereas for the bare Coulomb interaction, they don't.

In this article, we present a new self-consistent diagrammatic expansion for the electron self energy, in which the interaction lines are renormalized. The systematic perturbative expansion sums up the skeleton diagrams which exclude electron loops that represent a contribution to the charge fluctuations. Renormalization of the interaction lines is therefore achieved by the screening of the bare Coulomb interaction by the charge fluctuations. Although the approximation is exact, assuming that the model under the study is perturbative, only if the diagrams to all orders are included, it may converge rapidly if the screened interaction is considerably weaker than the bare Coulomb interaction. For the large-on-siteCoulomb-repulsion- $U$ 2-D Hubbard model, we evaluate the exact screened interaction from the QMC data, and show that this is indeed the case near, but sufficiently doped away from, half filling. Then, by using this exact screened interaction, we evaluate the electron self energy up to the third order, and compare to the QMC results. We find that the imaginary part of the self energy at low energies almost converges to the exact QMC results at the third order.

This article is organized as follows: In Section II we formulate our approximation. In Section III we present and discuss our results for the one-particle self energy along with the exact QMC and approximate FLEX calculations. We also calculate the exact QMC Fermi surfaces of the 2-D Hubbard model and discuss them in Section IV. And finally, we summarize our main findings in Section $\mathrm{V}$.

\section{METHOD}

We will develop our diagrammatic expansion for the electron self energy for the case of the 2-D one-band extended Hubbard model. The expansion is valid in other dimensions as well and is easily generalizable to multiband models. The Hamiltonian is

$$
\mathcal{H}=\sum_{i j}\left(-\sum_{\sigma} t_{i j} c_{i \sigma}^{\dagger} c_{j \sigma}+\frac{1}{2} \sum_{\sigma \sigma^{\prime}} V_{i j} n_{i \sigma} n_{j \sigma^{\prime}}\right),
$$

where $c_{i \sigma}^{\dagger}$ creates an electron with spin $\sigma$ at site $\mathbf{R}_{i}$ on an $N=L \times L$ square lattice with periodic boundary conditions, and $n_{i \sigma}=c_{i \sigma}^{\dagger} c_{i \sigma}$. The lattice constant, $a$, is set to be 1 . The Coulomb matrix element $V_{i j} \equiv V\left(\mathbf{R}_{i}-\mathbf{R}_{j}\right)$ comprises the Hubbard on-site repulsion $U \equiv V(\mathbf{0})$ and an extended part, $V(\boldsymbol{\Delta} \mathbf{R})$, for non-zero $\boldsymbol{\Delta} \mathbf{R}$. For the calculations done in this article, we include only a firstneighbor hybridization $t$ and the chemical potential $\mu$ in $t_{i j}$, and the on-site $U$ in $V(\boldsymbol{\Delta} \mathbf{R})$. For the hightemperature-superconducting cuprates, it is estimated that $t \sim 0.3-0.5 \mathrm{eV}$ and $U / t \sim 8-126$, $\mathrm{Ge}$ will use $U / t=8$ in all our calculations.

In Fig. 11(a), we show the generic form of the exact screened interaction, $V_{\mathrm{s}}$, expressed in terms of the bare Coulomb interaction, $V$, and the exact polarization insertion, $P$. In momentum-frequency space,

$$
V_{\mathrm{s}}(Q)=\frac{V(Q)}{1-P(Q) V(Q)},
$$

where $Q=(\mathbf{Q}, i \Omega)$. Fig. 1 (b) shows the zeroth- and firstorder diagram contributions to $P$, expressed in terms of $V_{\mathrm{s}}$. This form of expression is useful because it allows one to write down a self-consistent approximation for $V_{\mathrm{s}}$ and $P$, given a one-particle Green's function.

(a)

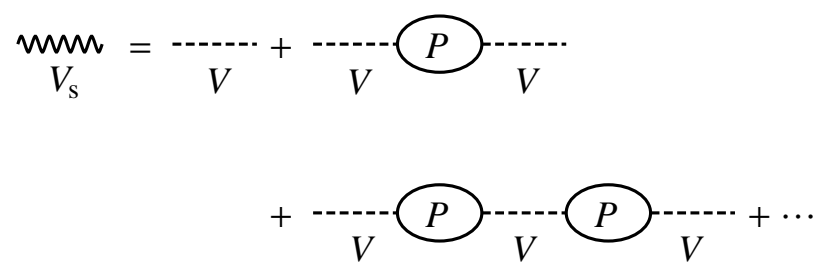

(b)

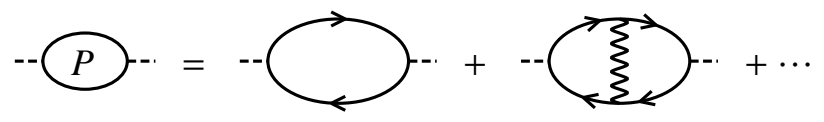

FIG. 1. (a) Exact screened interaction, $V_{\mathrm{s}} . V$ is the bare Coulomb interaction. (b) Exact polarization insertion, $P$.

These two simply-related quantities are also similarly related to a physical quantity easily measurable in QMC studies, the charge susceptibility, $\chi_{\mathrm{c}}$. The expression for this two-particle correlation function as a thermodynamic expectation value is

$$
\begin{aligned}
\chi_{\mathrm{c}}(\mathbf{Q}, i \Omega)=\frac{1}{N} \sum_{i j} \int_{0}^{\beta} d \tau & e^{i \Omega \tau-i \mathbf{Q} \cdot\left(\mathbf{R}_{i}-\mathbf{R}_{j}\right)} \\
& \times\left\langle\Delta n_{i}(\tau) \Delta n_{j}(0)\right\rangle,
\end{aligned}
$$


where $n_{i}(\tau) \equiv \sum_{\sigma} c_{i \sigma}^{\dagger}(\tau) c_{i \sigma}(\tau)$, and $\Delta n_{i}(\tau) \equiv n_{i}(\tau)-$ $\left\langle n_{i}(\tau)\right\rangle$.

In principle, the definition of a polarization insertion, $P$, is arbitrary, and so is of a screened interaction, $V_{\mathrm{s}}$, through Eq. (2). $P$ defined in this article through the diagrammatic expansion in Fig. 11(b) actually corresponds to the polarization insertion of the charge susceptibility, $\chi_{\mathrm{c}}$. The exact $\chi_{\mathrm{c}}$ is expressible as a geometric series in terms of the exact $P$, such that

$$
-\chi_{\mathrm{c}}(Q)=\frac{P(Q)}{1-P(Q) V(Q)}
$$

(see also Fig. 2). Combining Eqs. (2) and (4), we obtain an important expression for the screened interaction, $V_{\mathrm{s}}$, in terms of the physical charge susceptibility of the system, $\chi_{\mathrm{c}}$;

$$
\begin{gathered}
V_{\mathrm{s}}(Q)=V(Q)-V(Q) \chi_{\mathrm{c}}(Q) V(Q) . \\
-\chi_{\mathrm{c}}=P+P+P
\end{gathered}
$$

FIG. 2. The charge susceptibility, $\chi_{\mathrm{c}}$, expanded in terms of the polarization insertion, $P . V$ is the bare Coulomb interaction.

At this point, we are ready to calculate the exact $V_{\mathrm{s}}$ by using the exact $\chi_{\mathrm{c}}$ obtained by QMC. Using $V(Q)=U$ for the Hubbard model, we have,

$$
V_{\mathrm{s}}(Q)=U-U^{2} \chi_{\mathrm{c}}(Q) .
$$

One can also Fourier transform $V_{\mathrm{s}}(Q)$ to the real space, by using,

$$
V_{\mathbf{S}}(\boldsymbol{\Delta} \mathbf{R}, i \Omega)=\frac{1}{N} \sum_{\mathbf{Q}} e^{i \mathbf{Q} \cdot \mathbf{\Delta} \mathbf{R}} V_{\mathrm{s}}(\mathbf{Q}, i \Omega) .
$$

Again, for the Hubbard model,

$$
V_{\mathrm{s}}(\boldsymbol{\Delta} \mathbf{R}, i \Omega)=U \delta(\boldsymbol{\Delta} \mathbf{R})-U^{2} \chi_{\mathrm{c}}(\boldsymbol{\Delta} \mathbf{R}, i \Omega),
$$

where $\delta$ is the Kronecker delta. In Fig. 3, we plot the on-site and time-independent component of the exact screened interaction obtained from QMC, $V_{\mathrm{s}}(\boldsymbol{\Delta} \mathbf{R}=$ $\mathbf{0}, i \Omega=0$ ), for different hole dopings, $x \equiv 1-\langle n\rangle$, measured with respect to half filling. Although the bare (unscreened) Coulomb interaction, $U$, is equal to $8 t$, strikingly, the corresponding component of the exact screened interaction is substantially weaker for intermediate hole dopings, $x$, actually vanishing and changing sign, i.e., becoming attractive, at $x \sim 15 \%$. We will refer to this effect as "overscreening," 8 and explain below why it happens.

From Eq. (8), because of the $U^{2}$ term, one can suspect that for sufficiently large $U$, this term will win and

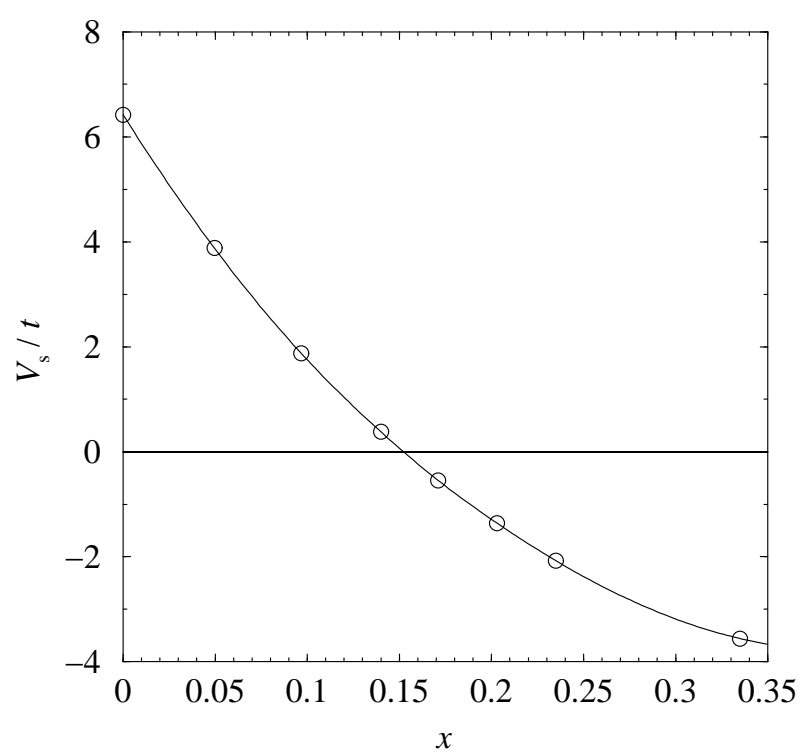

FIG. 3. $V_{\mathrm{s}}(\boldsymbol{\Delta} \mathbf{R}=\mathbf{0}, i \Omega=0)$ as a function of the hole doping, $x \equiv 1-\langle n\rangle$, obtained from QMC for the 2-D Hubbard model. Parameters are $U / t=8 ; T / t=1 / 3$, where $T$ is the temperature; and the lattice size is $8 \times 8$. 
$V_{\mathrm{s}}(\boldsymbol{\Delta} \mathbf{R}=\mathbf{0}, i \Omega=0)$ will become negative (attractive), since $\chi_{\mathrm{c}}(\boldsymbol{\Delta} \mathbf{R}=\mathbf{0}, i \Omega=0)$ is always positive and approaches a nonzero $U$-independent limit [of $\mathcal{O}(x / t)$ by a simple $U=\infty$ scaling argument] for $U \gg t$. On the other hand, at half filling, charge fluctuations are suppressed; so that $\chi_{\mathrm{c}}(\boldsymbol{\Delta} \mathbf{R}, i \Omega=0) \sim \mathcal{O}\left(t^{2} / U^{3}\right)$ and $V_{\mathrm{s}}(\boldsymbol{\Delta} \mathbf{R}=\mathbf{0}, i \Omega=0) \simeq U$, for $U \gg t$. Hence, presence of a sufficiently large $U$ and finite hole doping off half filling, $x$, is necessary for overscreening. Also, note that overscreening is not restricted to the on-site component of the Coulomb interaction; the nearest-neighbor components, and so forth, can also become attractive because of the overscreening effect 8

At this point, we are ready to write down a controlled perturbative expansion for the one-particle self energy. Since the exact screened interaction, $V_{\mathrm{s}}$, was shown to be substantially weaker than the bare interaction, the Hubbard $U$, one can expect that an expansion in terms of $V_{\mathrm{s}}$ should converge much more rapidly than a brute-force expansion in terms of $U$. As a matter of fact, it is not difficult to pick up the diagrams which contribute to this expansion. Keeping in mind that our approximation for the self energy will be self consistent in the sense that all one-particle-Green's-function lines will correspond to the full Green's function, we should allow only the skeleton diagrams in which none of these lines have any self-energy diagrams explicitly attached to them. As a second step, bearing in mind the fact that the interaction lines in our diagrams, which correspond to $V_{\mathrm{s}}$, already include the polarization diagrams in them [Fig. 1](a)], we omit any self-energy diagram in which an interaction line has a polarization diagram [see Fig. 1](b)] inserted. These are basically the only rules needed in the diagram selection. If all the diagrams to infinite order are included, an exact perturbation series will result, like in any other rigorous perturbative expansion. Note that the interaction lines in our diagrams, or $V_{\mathrm{s}}$, represent the bare Coulomb interaction, $U$, screened by the charge fluctuations, $\chi_{\mathrm{c}}$ [Eq. (5)]. Therefore, we have derived a diagrammatic expansion in terms of the charge fluctuations, which is exact if carried out to all orders. Moreover, because of the overscreening effect discovered by the analysis of the interaction lines $\left(V_{\mathrm{s}}\right)$ obtained by QMC exactly, a weak-coupling expansion to a first few orders is expected to converge in a rapid, controlled fashion.

In Fig. 1, we show all the self-energy diagrams up to the third order. The Hartree diagram in Fig. 1 (a) is written separately in terms of the bare interaction ( $U$ in this case) to prevent double counting; for the one-band Hubbard model, it is a trivial constant which is equal to $U\langle n\rangle$. The other first order diagram in Fig. 1(a) is like the Fock diagram with the bare interaction line replaced by the screened interaction. This diagram combines the bare-interaction Fock diagram (which is again equal to a trivial constant, $-U\langle n\rangle / 2$, in this case) with the chargefluctuation self energy diagram found in FLEX-like approximations; because, the screened interaction is the sum of the bare interaction and the charge-fluctuation

propagator [see, e.g., Eq. (5) or (6)]. In the second order, there is only one diagram contributing to the self energy [Fig. A(b)]; and in the third order the number of all the contributing diagrams is six [Fig. $1(\mathrm{c})]$.

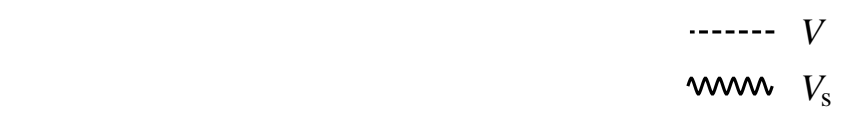

(a)

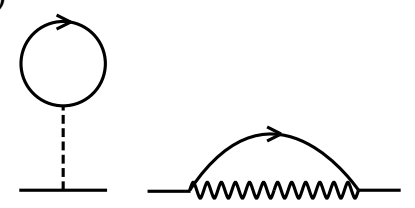

(b)

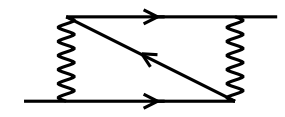

(c)
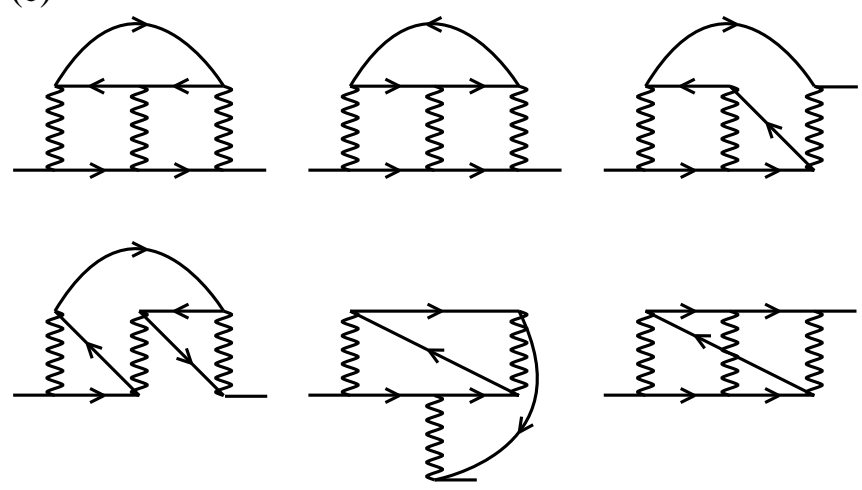

FIG. 4. All the contributing self energy diagrams in the first (a), second (b), and third order (c) of the screened-interaction $\left(V_{\mathrm{s}}\right)$ expansion. The weak-coupling expansion would become exact (assuming the model under study is perturbative) when carried out to all orders.

At this point, we would like to discuss the fact that, the screened-interaction expansion developed here is a low-energy approximation. In Fig. 同, we plot the exact screened interaction, $V_{\mathrm{s}}(\mathbf{Q}, i \Omega)$, which was obtained by $\mathrm{QMC}$, as a function of the frequency, $\Omega$. For the chosen $\mathbf{Q}$ point, $V_{\mathrm{s}}$ is actually attractive and small at zero frequency, and vanishes at a slightly higher frequency. But as we approach the first Matsubara frequency, $V_{\mathrm{s}}$ rapidly grows, and it is already more than half of the bare, unscreened Coulomb repulsion. As the frequency increases further, $V_{\mathrm{s}}$ finally approaches to the bare repulsive interaction, $U=8 t$. It is now clear that the high-energy part of the $V_{\mathrm{s}}$ is not weak, and our approximation is limited to the low-energy scales. Therefore, for the temperature studied here $(T=t / 3)$, the calculated self energy is expected to be accurate only for the first Matsubara frequency, $\pi T \approx t$, a small-enough fraction of the width of the tight-binding band, which governs the energy scale for $V_{\mathrm{s}}$. 


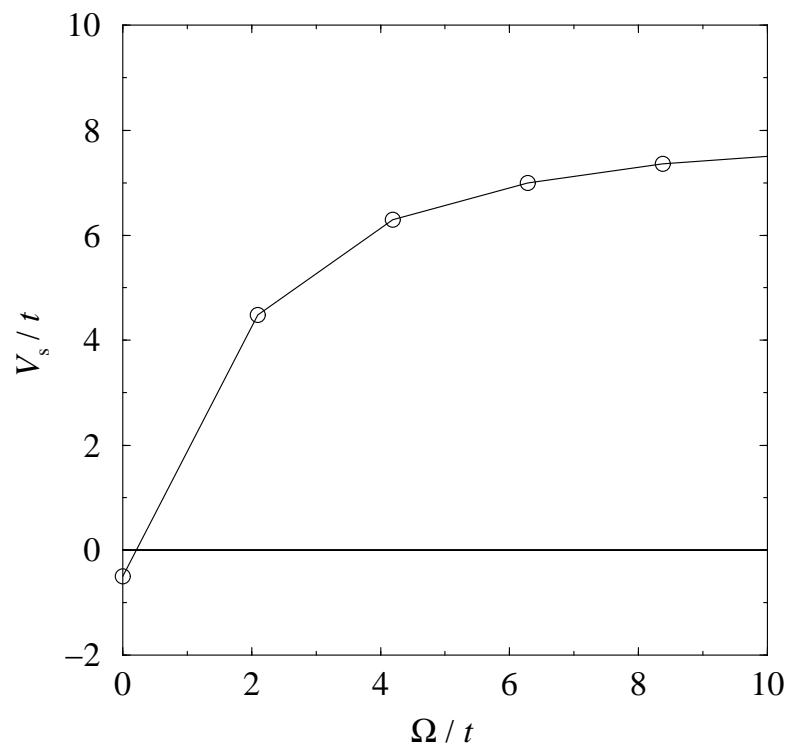

FIG. 5. The exact screened interaction, $V_{\mathbf{s}}(\mathbf{Q}, i \Omega)$, calculated by $\mathrm{QMC}$, shown as a function of the frequency, $\Omega$. The momentum point is $\mathbf{Q}=(\pi / 2,0)$ on an $8 \times 8$ lattice. Parameters are $U / t=8 ; T / t=1 / 3$, where $T$ is the temperature; and $x=14.0 \%$, where $x \equiv 1-\langle n\rangle$.

Before finishing this section, we would like to discuss a peculiar property of the irreducible polarization insertion and the associated three-point vertex function. As we discussed earlier, the exact screened interaction, $V_{\mathrm{s}}$, as obtained from QMC, vanishes at particular $(\boldsymbol{\Delta} \mathbf{R}, i \Omega)$ points, and it will vanish at particular $(\mathbf{Q}, i \Omega)$ points likewise; this was the motivation behind developing a weak-coupling expansion in terms of $V_{\mathrm{s}}(Q)$. But from Eq. (2), $V_{\mathrm{s}}(Q)=0$ immediately implies that $P(Q)=\infty$ at the same $Q=(\mathbf{Q}, i \Omega)$ point. This divergence in the polarization insertion of the charge susceptibility does not imply a divergence in the charge susceptibility itself, which is the actual physical quantity. In fact, from Eq. (四), $P(Q)=\infty$ simply implies that $\chi_{\mathrm{c}}(Q)=1 / V(Q)$. Although this divergence is not a worry from a physics point of view, it imposes technical difficulties on the expansion of $P(Q)$ [see Fig. 1 (b)] along these singularities; to overcome this difficulty in an actual calculation of $P(Q)$, it would be more appropriate to expand its inverse, $1 / P(Q)$, rather than to expand $P(Q)$ itself directly.

Both the polarization insertion and the self energy can be expressed in terms of a three-point irreducible vertex function, $\Lambda(Q, k)$, by the exact relations (see Fig. 6),

$$
P(Q)=\frac{2 T}{N} \sum_{k} G(k) G(k-Q) \Lambda(Q, k)
$$

and

$$
\Sigma(k)=V(Q=0)\langle n\rangle-\frac{T}{N} \sum_{Q} \Lambda(Q, k) V_{\mathrm{s}}(Q) G(k-Q),
$$

where $G(k)$ is the one-particle Green's function and $k=$ $(\mathbf{k}, i \omega)$. Eq. (9) immediately implies that for a given $Q$, if $P(Q)=\infty$, then $\Lambda(Q, k)=\infty$, because $G(k)$ is always finite. Even though $\Lambda(Q, k)$ in Eq. (10) may diverge, the one-particle self energy, $\Sigma(k)$, which is the actual physical quantity, remains finite; because, if $\Lambda(Q, k)$ diverges at a given $Q$, then $V_{\mathrm{s}}(Q)$ will vanish, therefore canceling the divergence. But, there is still a concern that a weakcoupling expansion for $\Lambda$ in Fig. 6(b), which generates the diagrams in Fig. 1, may not be sufficient because of this diverging behavior. We will put our theory to numerical testing in the next section.

(a)

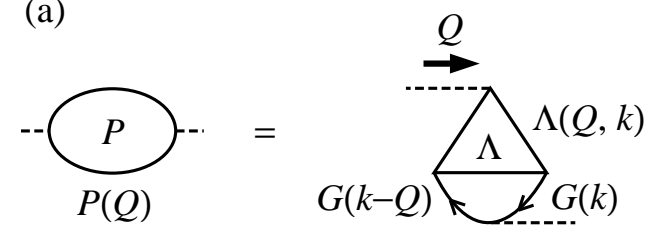

(b)

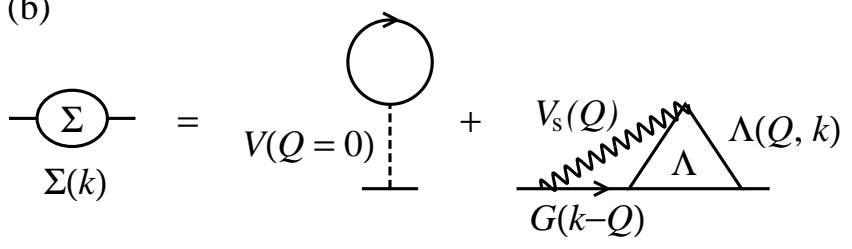

FIG. 6. The polarization insertion, $P(Q)$ (a), and the one-particle self energy, $\Sigma(k)(\mathrm{b})$, in terms of the three-point irreducible vertex, $\Lambda(Q, k)$. $G(k)$ is the one-particle Green's function, $Q=(\mathbf{Q}, i \Omega)$, and $k=(\mathbf{k}, i \omega) . V$ and $V_{\mathbf{s}}$ are the bare and the screened interaction respectively.

\section{ONE-PARTICLE SELF ENERGY}

We perform our numerical calculations for the self energy on periodic discreet lattices of sizes $4 \times 4$ and $8 \times 8$, so that they can be compared with the QMC results. We use the Matsubara-frequency representation, and employ a fermion frequency cutoff of $\sim 35 t$. All the QMC calculations were done at a temperature, $T=t / 3$, and with the cutoff employed here, this corresponds to 16 positive Matsubara frequencies. Note that QMC calculations are performed in the imaginary time, and a time discretization is made for numerical purposes. The number of time 
slices in $[0, \beta=1 / T]$ used in the QMC calculations was 80 , which is equivalent to using 40 positive Matsubara frequencies - even larger a cutoff than our diagrammatic calculations. We chose a smaller cutoff for our diagrammatic calculations to avoid the high-frequency errors in the $V_{\mathrm{s}}$ obtained from QMC, which goes directly into the calculations. We vary the chemical potential, $\mu$, in order to match the filling, $\langle n\rangle$, with that of the QMC. In order to calculate $\langle n\rangle$, the one-particle Green's function, $G(\mathbf{k}, i \omega)$, has to be summed over the Matsubara frequencies, and to prevent cutoff effects in this relatively-slowconverging sum, we effectively extend the sum to infinity by using the standard - replace the high-frequency part of the sum with the result for the non-interacting casetrick.

In Fig. 17, we plot the real and imaginary parts of the self energy as a function of Matsubara frequencies along with the results from QMC calculations. We chose a particular momentum point close to the Fermi surface, $\mathbf{k}=(\pi, 0)$, but the $\mathbf{k}$ dependence of both the QMC and the screened-interaction-expansion calculations near the Fermi surface is small. QMC error bars are omitted in all but one plot in this article for clarity, but the estimated QMC error bar for the real and imaginary parts of the self energy at the first frequency is about $\pm 0.1 t$. The corresponding error bar for the QMC Green's function is about $\pm 0.02 t^{-1}$. Also, the error bars for the self energy grow with the frequency, whereas, they are roughly constant for the Green's function. Note that, the temperature in these calculations is $T=t / 3$, and the second Matsubara Frequency, $5 \pi T$, is already about $5.2 t$, which is a substantial fraction of the (non-interacting) bandwidth of our model, $8 t$. For the reasons we explained in the previous section, our approximation is a low-energy approximation, which is unable to capture this regime. But, the first Matsubara frequency, $\pi T \approx 1.0 t$, is low enough compared to the bandwidth, and remarkably, at the $14 \%$ hole doping, where the screened interaction almost vanishes (see Fig. 3), our approximation for the imaginary part of the self energy almost converges to the QMC result within the error bar [Fig. [(b)]. This shows that our expansion may indeed be converging vary rapidly at the hole doping at which the on-site component of the screened interaction at zero frequency, $V_{\mathrm{s}}(\boldsymbol{\Delta} \mathbf{R}=\mathbf{0}, i \Omega=0)$, vanishes. But, when the hole doping is varied away from this point, $\operatorname{Im} \Sigma$ varies in opposite direction with respect to the exact QMC results. Naturally, having the correct doping dependence is one of the most desired aspect of any approximation, which, therefore, substantially limits the usefulness of this expansion. It is quite possible that the reason for this failure may be intimately related to the vertex divergence problem discussed in the previous section. In Fig. 8, we show the evolution of the self energy with the order of the diagrams included. The imaginary part, in fact, seems to be converging to the QMC result at the lowest frequency with the increasing order, and it is about the same as the QMC result within the QMC error bar (see Fig. 10) at the third order. But the real
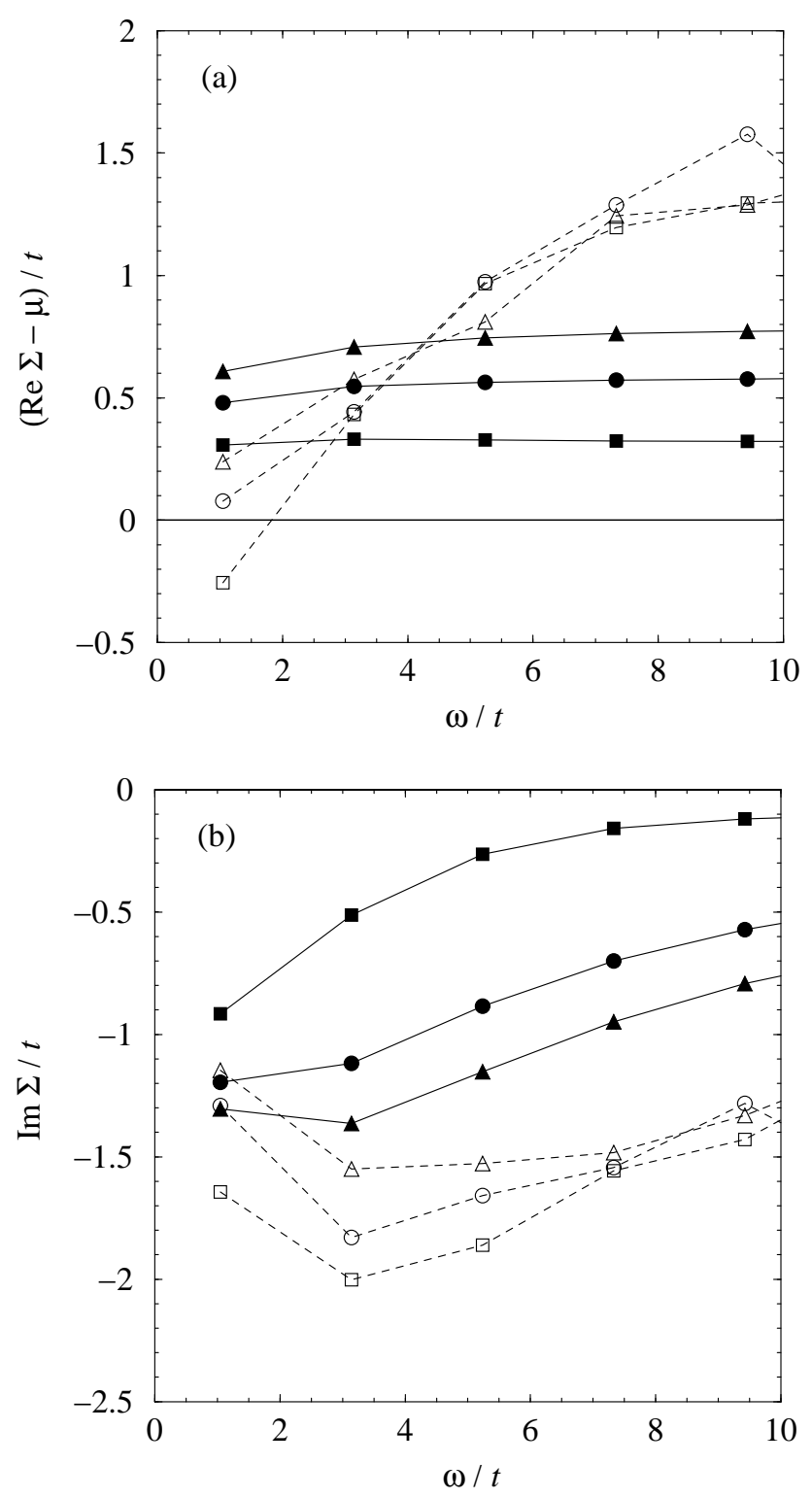

FIG. 7. The one-particle self energy as a function of the Matsubara frequency, shown for the screened-interaction expansion (solid lines and filled symbols) along with exact (except for statistical errors) QMC results (dashed lines with empty symbols). The $\mathbf{k}$ point is $(\pi, 0)$. Hole dopings, $x \equiv 1-\langle n\rangle$, are 10.0 (squares), 14.2 (circles), and $17.5 \%$ (triangles). Other parameters are $U / t=8$ and $T / t=1 / 3$. Lattice sizes are $4 \times 4$ for the screened-interaction expansion and $8 \times 8$ for QMC. Hole dopings for both the expansion and the QMC correspond to averages on $4 \times 4 \mathrm{k}$-space meshes. Actual QMC hole dopings averaged on $8 \times 8 \mathbf{k}$-space meshes are $9.7,14.0$, and $17.1 \%$. The real (a) and the imaginary (b) part. [We use $8 \times 8 \mathrm{QMC}$ results in these comparisons because QMC calculations were performed only on $8 \times 8$ lattices for most hole dopings; but the difference between $4 \times 4$ and $8 \times 8$ QMC results are well within the error bars (see Fig. 10 and the associated discussion at the end of this section).] 
part seems to be going in the wrong direction with the increasing order.

The real part of the self energy which was calculated by the screened-interaction expansion actually does not do very well against the exact QMC results [Fig. 7(a)] at all, but, as we will analyze below, this is not a failure of the expansion itself. In fact, this is merely the quantity which determines the location of the Fermi surface. Luttinger theorem 9 states that, for any diagrammatic expansion, the total volume (area in 2-D) enclosed by the Fermi surface is equal to the total filling, $\langle n\rangle$; therefore our approximation will obey this statement. But then, this simply implies that the exact QMC results may not obey the Luttinger theorem for the volume of the Fermi surface. To investigate this further, we calculated the exact QMC Fermi surfaces for various dopings, which will be discussed later.

Fig. 9 shows results for the FLEX calculations in comparison with the QMC. FLEX approximation employed here uses exchange of charge and spin fluctuations, i.e., the particle-hole-channel, but omits the particle-particle fluctuations (for the Hubbard model there would only be the particle-particle fluctuations of the singlet type). The results for the real part are very similar to the screened-interaction expansion. FLEX has a somewhat better qualitative behavior at high frequencies. Low frequency behavior of the real part for FLEX and screenedinteraction expansions are expected to be very similar because they are both expected to obey the Luttinger theorem. The real part for FLEX at the lowest frequency is doing somewhat worse than the screened-interaction expansion, probably because $\operatorname{Im} \Sigma$ for FLEX is somewhat bigger, indicating that FLEX results are more distant to their zero- or low-temperature values [compare Figs. 1](b) and 9(b)]. Im $\Sigma$ is expected to vanish at zero temperature and zero frequency also according to the Luttinger theorem. FLEX results for the $\operatorname{Im} \Sigma$ show a correct qualitative behavior at high frequencies. Although they also capture the doping dependence correctly, the magnitude of the doping dependence is smaller by an order. In addition, they don't do well quantitatively at any particular doping.

The screened-interaction-expansion calculations performed in this article are computationally very time consuming. The reason for this is the requirement for the summation over many frequency and momentum variables for the third and higher order diagrams. In order to go to the fourth order, one would probably need Monte Carlo summation techniques. Even at the third order, we were able to perform our calculations on $4 \times 4$ lattices only. In Fig. 10 we make some comparisons of the calculations on different lattice sizes. $4 \times 4$ and $8 \times 8$ QMC results are compared to the screened-interactionexpansion results. FLEX results are shown as well. The difference between the results for the two lattice sizes seems to be very small. Estimated QMC error bars for the $\operatorname{Im} \Sigma$ on the $8 \times 8$ lattice are also shown. These are the approximate upper bounds to the statistical errors
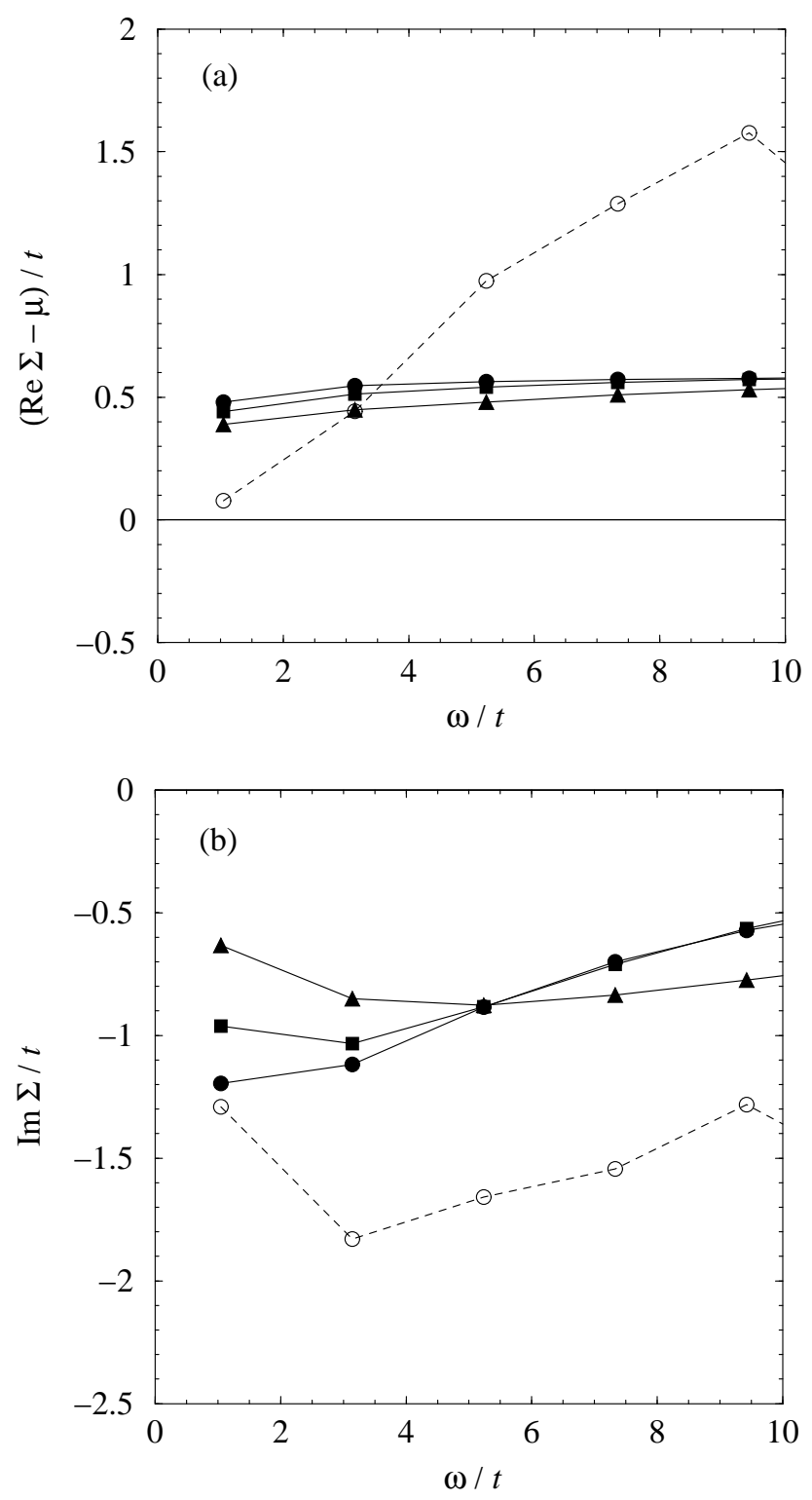

FIG. 8. The one-particle self energy as a function of the Matsubara frequency, shown for the 1st- (triangles), 2nd(squares), and 3rd-order (circles) screened-interaction expansion (solid lines and filled symbols) along with exact (except for statistical errors) QMC results (dashed lines and empty circles). The $\mathbf{k}$ point is $(\pi, 0)$. Hole doping, $x \equiv 1-\langle n\rangle$, is $14.2 \%$. All parameters are the same as in Fig. 1. The real (a) and the imaginary (b) part. 
associated with the QMC calculations.
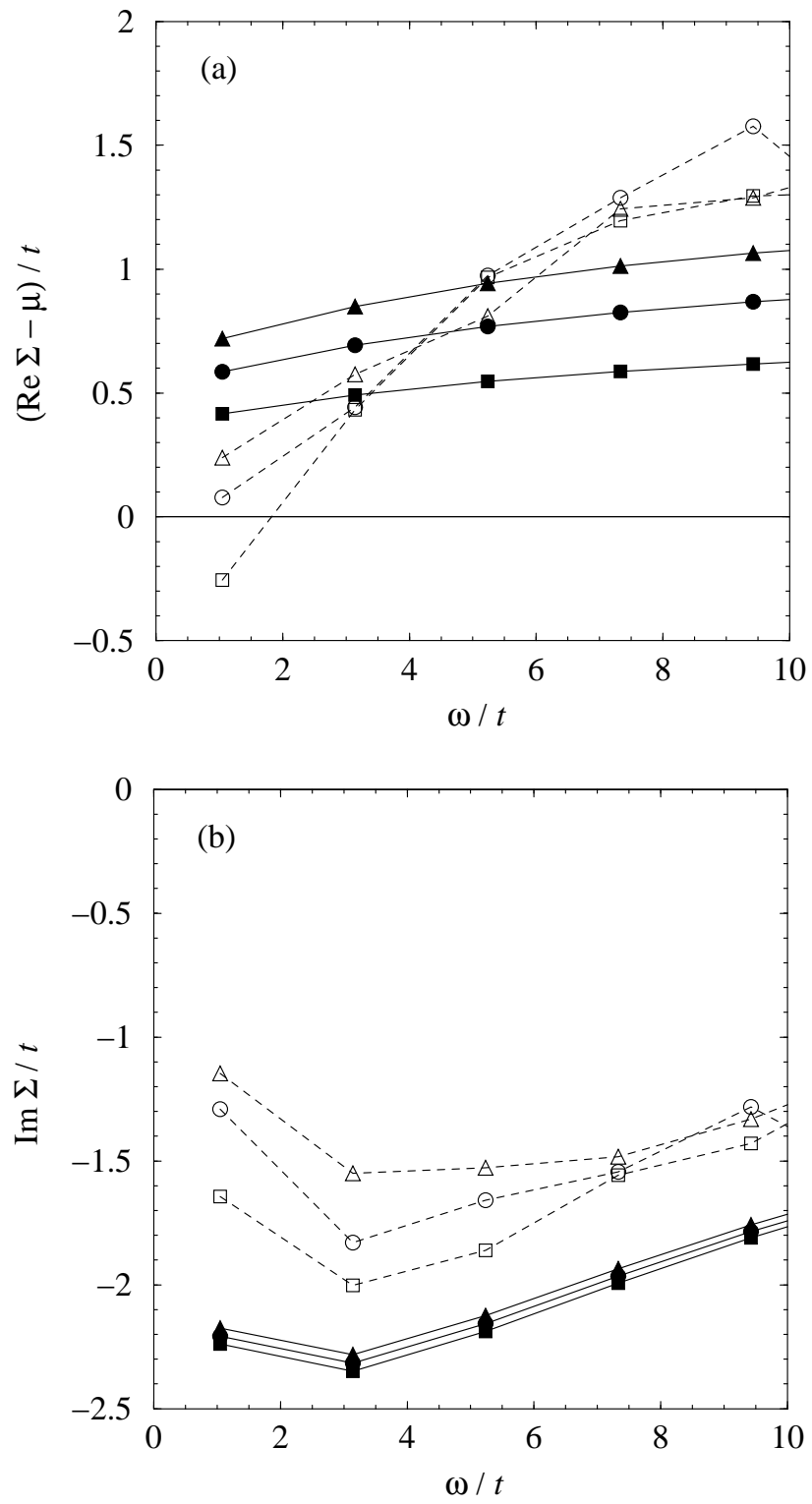

FIG. 9. The one-particle self energy as a function of the Matsubara frequency, shown for FLEX approximation (solid lines and filled symbols) along with exact (except for statistical errors) QMC results (dashed lines with empty symbols). The $\mathbf{k}$ point is $(\pi, 0)$. Hole dopings, $x \equiv 1-\langle n\rangle$, are 10.0 (squares), 14.2 (circles), and $17.5 \%$ (triangles). Other parameters are $U / t=8$ and $T / t=1 / 3$. Lattice sizes are $4 \times 4$ for FLEX and $8 \times 8$ for QMC. Hole dopings for both the FLEX and the QMC correspond to averages on $4 \times 4 \mathrm{k}$-space meshes. Actual QMC hole dopings averaged on $8 \times 8 \mathrm{k}$-space meshes are $9.7,14.0$, and $17.1 \%$. The real (a) and the imaginary (b) part.

\section{EXACT QMC FERMI SURFACE}

In this section, we will discuss the exact QMC Fermi surfaces calculated. Although there are several equivalent definitions for the Fermi surface, the definition which locates the Fermi surface as the zero frequency poles of the Green's function is most suitable for finite temperatures. One finds the solution of $\varepsilon(\mathbf{k})+\operatorname{Re} \Sigma(\mathbf{k}, \omega=0)=\mu$ in order to locate such poles. 6 For a Fermi liquid, these poles correspond to the quasiparticles at the Fermi surface. But, the definition is general, and can also be used at finite temperatures. Note that, the Luttinger theorem, which relates the volume enclosed by the Fermi surface to the total electron filling, is strictly valid only at zero temperature; but, as long as the temperature is not unreasonably high, one finds that the Luttinger theorem is satisfied in an approximate manner, and the only change to the quasiparticle picture is some temperature broadening due to the finite $\operatorname{Im} \Sigma(\mathbf{k}, \omega=0)$. As mentioned in the previous section, the quantity, $\operatorname{Im} \Sigma(\mathbf{k}, \omega=0)$, is indeed a good indication of how close one is to $T=0$, which should actually vanish at $T=0$ for a system obeying the Luttinger theorem. In order to numerically calculate the Fermi surface on a finite $8 \times 8$ QMC lattice, we first find the solution of $\varepsilon(\mathbf{k})+\operatorname{Re} \Sigma(\mathbf{k}, \omega=0)=\mu$ by interpolating between the $\mathbf{k}$ points. Moreover, $\Sigma(\mathbf{k}, \omega=0)$, which is very close in value to the one at the lowest available frequency, $\Sigma(\mathbf{k}, \omega=i \pi T)$, is found by linear extrapolation using the lowest-two frequencies. Due to this numerical approximation procedures, the systematic interpolation and extrapolation errors up to a few percent are possible.

Fig. 11(a) shows the exact QMC Fermi surfaces for actual hole dopings, $x \equiv 1-\langle n\rangle$, ranging from 5.0 to $33.5 \%$. These actual hole dopings in decreasing order are $x=33.5$ [smallest surface centered around $\mathbf{k}=(0,0)$ ], $23.5,20.3,17.1,14.0,9.7$, and $5.0 \%$ (the arcs centered around the Brillouin-zone corners). We found the areas enclosed by the Fermi surfaces by numerical integration, and calculated the hole dopings implied by the Luttinger theorem corresponding to these areas. Note that, due to the curve-fitting procedures employed for the discrete $(\mathbf{k}, \omega)$ space, these areas are uncertain within a few percent. The results in the same order as the actual hole dopings are $33.5,20.5,14.1,7.1,-4.3,-19.8$, and $-32.8 \%$. We plot these values against the actual doping in Fig. 11(b). Considering the fact that we are at a moderately high temperature, $T=t / 3$, and accounting for the curve-fitting errors, the Luttinger theorem seems to be satisfied at least qualitatively for hole dopings, $x \geq 17 \%$, i.e., the so-called overdoped region in the context of high-temperature superconductivity. On the other hand, in the underdoped region, QMC Fermi surfaces start deviating from the Luttinger theorem in a systematic qualitative matter. As one proceeds towards 

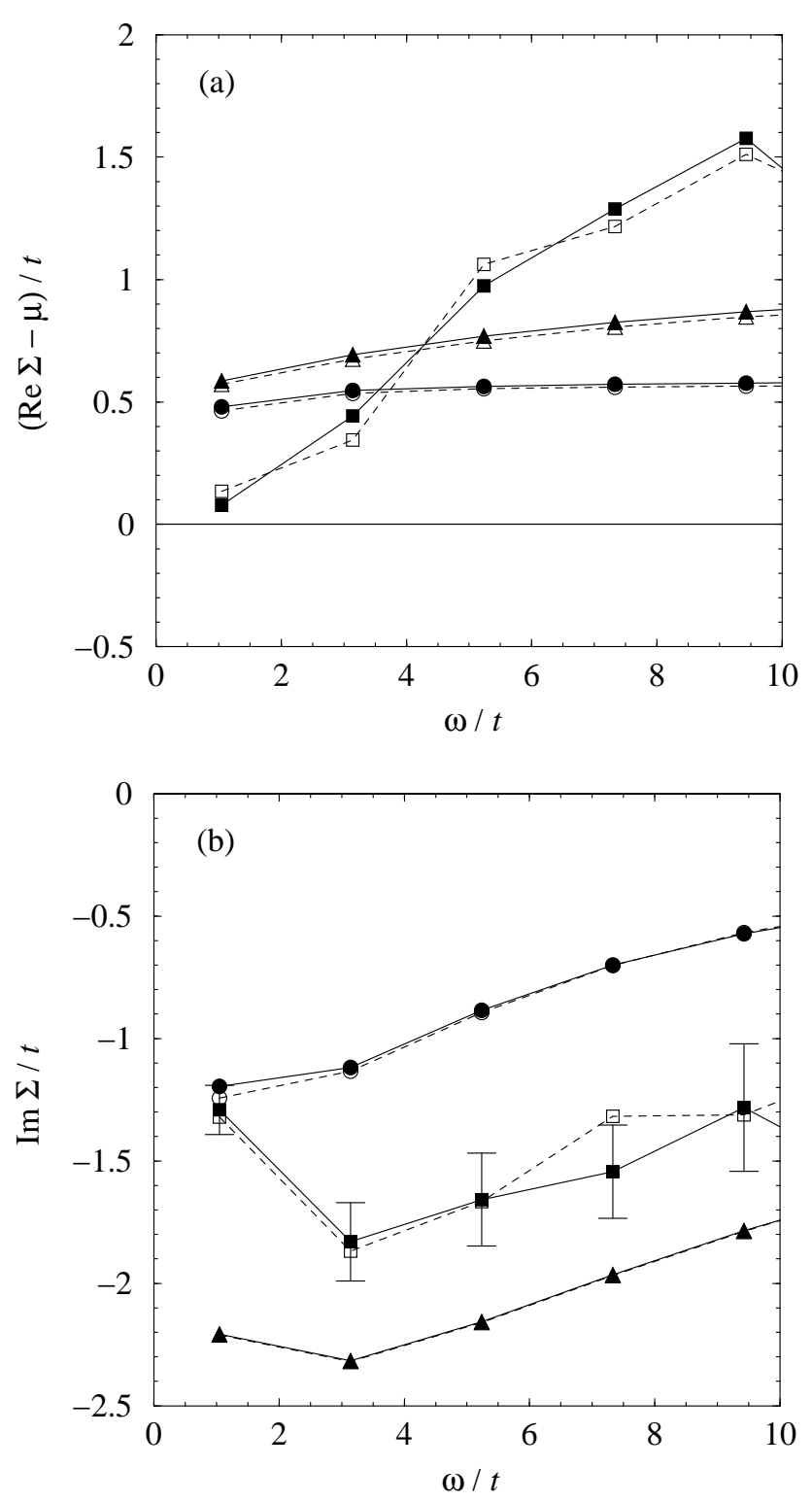

FIG. 10. The one-particle self energy plotted as a function of the Matsubara frequency for the purpose of evaluating lattice-size effects. Results for QMC (squares), screened-interaction expansion (circles), and FLEX approximation (triangles) are shown. The $\mathbf{k}$ point is $(\pi, 0)$. Solid lines and filled symbols represent $8 \times 8$ lattices for QMC and $4 \times 4$ otherwise. Dashed lines and empty symbols represent $4 \times 4$ lattices for all. Parameters are $U / t=8$ and $T / t=1 / 3$. Hole dopings, $x \equiv 1-\langle n\rangle$, averaged on $4 \times 4 \mathbf{k}$-space meshes are $14.2 \%$ (solid lines and filled symbols) and $13.8 \%$ (dashed lines and empty symbols). Actual, $8 \times 8$-mesh averaged hole doping for the $8 \times 8 \mathrm{QMC}$ lattice is $14.0 \%$ (solid line with filled squares). Note that the $4 \times 4$-mesh averaged hole-doping values for all data sets match each other among the solid and the dashed lines. The real (a) and the imaginary (b) part. Also shown are the QMC error bars for the $\operatorname{Im} \Sigma$ on the $8 \times 8$ lattice. half filling, Fermi surface starts shrinking towards the $\mathbf{k}=(\pi, \pi)$ point, looking like an electron-doped system. This is completely inconsistent with the concepts of the band theory and Fermi liquid. Fermi surface of the Hubbard model gradually gets smaller and disappears when the hole doping gets smaller in the underdoped region. Therefore, this seems to be a doping induced transition between a Fermi-liquid metal and a strongly-correlated insulator. This extreme violation of the Luttinger theorem may imply that there is no asymptotically convergent diagrammatic expansion for the Hubbard model in the underdoped region. But, it looks like, as the hole doping increases past the optimal doping $(\sim 15 \%)$, the system looks more like a conventional Fermi liquid, and diagrammatic expansions seem more likely to be possible. In Fig. 11(b), we plot the hole dopings, $x$, deduced from the area enclosed by the Fermi surface according to the Luttinger theorem against the actual hole dopings. Systematic deviation towards half filling is clearly seen. Note that the line is only a guide to the eye; it is quite possible that the area of the QMC Fermi surface at dopings very close to half filling gets vanishingly small. We don't have data for such dopings at the point, so, further investigation is nefsssary to determine the existence of such "hole pockets." 10 In order to rule out temperature effects one might also study lower temperatures, which could be accessible for such dopings very close to half filling. This figure also shows the results for FLEX calculations at the same temperature. Since FLEX is expected to satisfy the Luttinger theorem at low temperatures, the deviations in this case are known to be mainly because of temperature effects. But, these deviations are much more reasonable, and the FLEX results show approximately correct slope for all dopings. Doping deduced from the Luttinger theorem for FLEX calculations also have the correct sign for all dopings. We show the FLEX Fermi surfaces at $5.0 \%$ doping for the temperature studied above $(T=t / 3)$ along with a low-temperature Fermi surface in Fig. 12. The low- and intermediate-temperature Fermi surfaces for FLEX look very similar, and the low-temperature case is almost identical to the noninteracting case (for the Hubbard model, which is also the same as the HartreeFock case). The comprehensive results we obtained for the Fermi surface are also qualitatively consistent with the other recent QMC study of the Hubbard and hightemperature expansion study of $t$ - 11 models.

\section{SUMMARY AND CONCLUSIONS}

In this article, we studied a new approximation for the one-particle self energy of the large- $U$ Hubbard-like models. An expansion was written out in terms of a screened interaction, which is nonlocal and retarded, although it acts between local and instantaneous charge densities. The screening is achieved by the charge fluctuations. The expansion is an exact perturbative expansion, although 

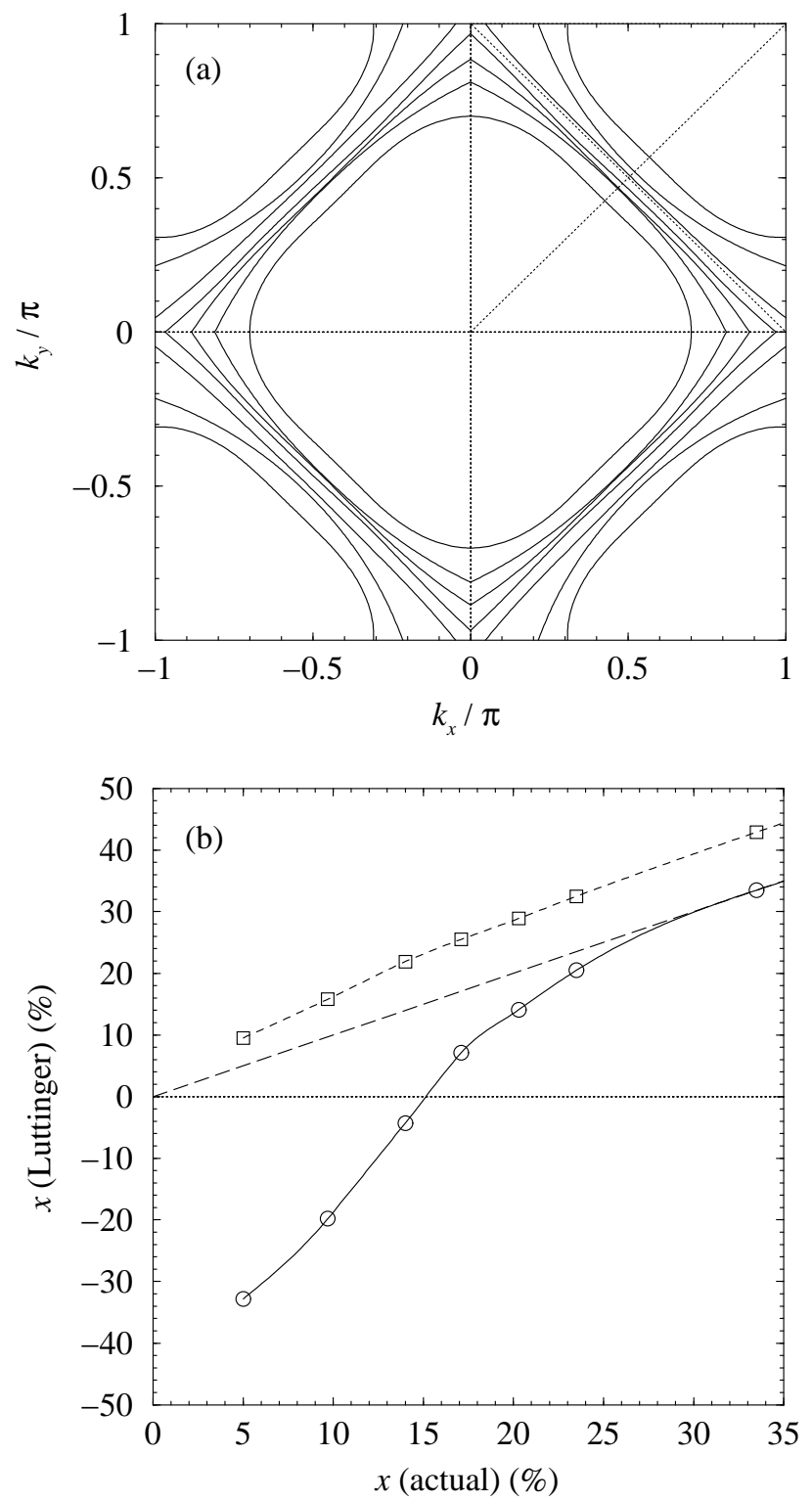

FIG. 11. (a) The exact QMC Fermi surfaces for various hole dopings. Parameters are $U / t=8$ and $T / t=1 / 3$. Actual hole dopings, $x \equiv 1-\langle n\rangle$, in decreasing order are $x=33.5$ [smallest surface centered around $\mathbf{k}=(0,0)$ ], 23.5, $20.3,17.1,14.0,9.7$, and $5.0 \%$ (the arcs centered around the Brillouin-zone corners). The lattice size is $8 \times 8$. (b) The hole dopings, $x$, deduced from the areas enclosed by the Fermi surfaces according to the Luttinger theorem, plotted against the actual hole dopings, $x \equiv 1-\langle n\rangle$ (circles and the solid line). Also shown are the FLEX results at the same temperature (squares and the dashed line). The long-dashed line without symbols corresponds to the Luttinger theorem. The lattice size for the FLEX calculations is $16 \times 16$.

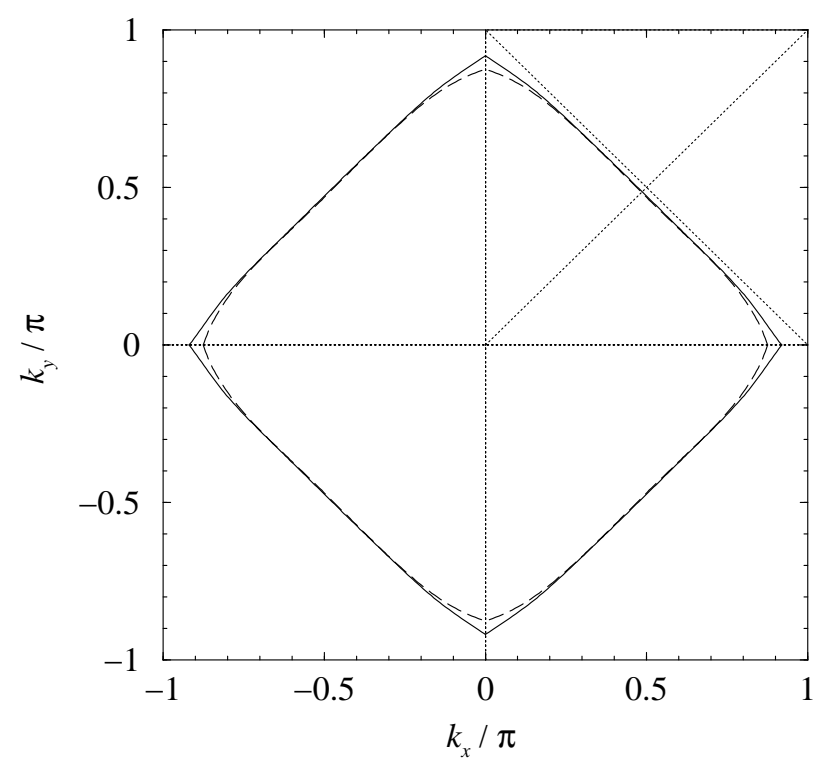

FIG. 12. The FLEX Fermi surface at the temperature $T / t=1 / 3$ (long-dashed line) and at a lower temperature, $T / t=1 / 16$ (solid line). This is the lowest available temperature for the lattice size studied $(16 \times 16)$ at this doping. Parameters are $U / t=8$ and $x \equiv 1-\langle n\rangle=5.0 \%$. Hole dopings, $x$, deduced from the areas according to the Luttinger theorem are $7.6 \%(T / t=1 / 16)$ and $9.5 \%(T / t=1 / 3)$. Note that the low-temperature FLEX Fermi surface is almost the same as the noninteracting (or Hartree-Fock) Fermi surface (not shown). 
this doesn't necessarily mean that it is an exact solution because of the possibility of nonperturbative nature of the large- $U$ systems. We carried out the expansion up to the third order. The screened interaction was determined by QMC calculations exactly, and then directly put into the expansion calculations. The local component of the exact screened interaction, which screens the bare Hubbard $U$, was found to be vanishing at certain small frequencies at hole dopings near $15 \%$. This is a very encouraging behavior for a weak-coupling expansion, which indicates that such expansions might be possible at lowenough frequencies where the screened-interaction is very small. However, the analysis of the three-point-vertex function, which, together with the screened interaction, determines the one-particle self energy, showed that it diverges at the same frequency-momentum points where the screened interaction vanishes. The expansion for the self energy had only limited success against the exact QMC results. Although the expansion gave excellent results for the low-energy part of $\operatorname{Im} \Sigma$ near $15 \%$ hole doping, where the screened interaction vanishes, it had the wrong doping dependence around this same value. Moreover, the real part of the QMC results were not consistent with a diagrammatic expansion which should obey the Luttinger theorem, although, we can't rule out the possibility of temperature effects. If it were because of temperature effects, this would also mean that our approximation doesn't work for Re $\Sigma$ at all, since with the increasing order, results for the expansion moved in the wrong direction. In contrast, $\operatorname{Im} \Sigma$ showed a converging behavior near $15 \%$ doping, as mentioned above. We also calculated FLEX self energies, which had the correct qualitative doping dependence and high-frequency behavior, but did actually worse quantitatively near $15 \%$ doping.

In order to investigate the QMC Re $\Sigma$ results, we numerically calculated the exact QMC Fermi surfaces. The results showed extreme violation of the Luttinger theorem near half filling, which might be because of a dopinginduced metal-insulator transition arising from strongcoupling effects. The Fermi surface of the Hubbard model seemed to be reducing to hole pockets centered around $\mathbf{k}=(\pi, \pi)$ at small hole dopings near half filling. Further QMC investigation at smaller hole dopings and lower temperatures might bring more evidence into this.

As far as the high-temperature superconductors are concerned, exact QMC results might be indicating that the underdoped region of these materials is a transition between a strong-coupling Mott-Hubbard insulator and a Fermi metal. The disappearance of the Fermi surface with the decreasing hole doping can also be viewed as an opening of a pseudogap.12 For the full investigation of this region at low temperatures, other nonperturbative treatments might be necessary. However, the partial success of the screened-interaction expansion developed here shows that it might be useful near optimal doping, as well as other diagrammatic expansions in the optimallydoped and overdoped regions.

\section{ACKNOWLEDGMENTS}

G. E. acknowledges useful discussions with Richard T. Scalettar and Andrew K. McMahan. This work was partially supported by National Science Foundation under the Grants No. DMR-92-15123 and No. DMR-9970291. Work at University of California, Davis was supported in part by an Accelerated Strategic Computing Initiative Grant and by Materials Research Institute of Lawrence Livermore National Laboratory. Computing support from University Computing and Networking Services at University of Georgia is gratefully acknowledged.

* Present address: Department of Physics, University of California, Davis, California 95616-8677; and Lawrence Livermore National Laboratory, University of California, Livermore, California 94550-9234

$\dagger$ E-mail address: esirgen@ucdavis.edu

¥ Present address: Institut für Theoretische Physik, Technische Universität Graz, 8010 Graz, Austria

${ }^{1}$ E. Dagotto, Rev. Mod. Phys. 66, 763 (1994).

${ }^{2}$ M. Imada, A. Fujimori, and Y. Tokura, Rev. Mod. Phys. 70, 1039 (1998).

${ }^{3}$ W. Fettes, I. Morgenstern, and T. Husslein, Computer Physics Communications 106, 1 (1997); H. Q. Lin, J. E. Hirsch, and D. J. Scalapino, Phys. Rev. B 37, 7359 (1988).

${ }^{4}$ C. Gröber, R. Eder, and W. Hanke, Phys. Rev. B 62, 4336 (2000).

${ }^{5}$ G. Esirgen, H.-B. Schüttler, and N. E. Bickers, Phys. Rev. Lett. 82, 1217 (1999); and references therein.

${ }^{6}$ M. S. Hybertsen, E. B. Stechel, M. Schlüter, and D. R. Jennison, Phys. Rev. B 41, 11068 (1990); C.-X. Chen and H.-B. Schüttler, ibid. 43, 3771 (1991); S. B. Bacci, E. R. Gagliano, R. M. Martin, and J. F. Annett, ibid. 44, 7504 (1991).

${ }^{7}$ H.-B. Schüttler and A. J. Fedro, Phys. Rev. B 45, 7588 (1992).

${ }^{8}$ H.-B. Schüttler, C. Gröber, H. G. Evertz, and W. Hanke, cond-mat/9805133; 0104300 (to be published).

9 J. M. Luttinger, Phys. Rev. 119, 1153 (1960).

${ }^{10}$ A. P. Kampf and J. R. Schrieffer, Phys. Rev. B 42, 7967 (1990); S. A. Trugman, Phys. Rev. Lett. 65, 500 (1990); D. Duffy and A. Moreo, Phys. Rev. B 51, 11882 (1995).

${ }^{11}$ W. O. Putikka, M. U. Luchini, R. R. P. Singh, Phys. Rev. Lett. 81, 2966 (1998).

${ }^{12}$ For experimental evidence of the pseudogap, see, e.g., the following and references therein: Z.-X. Shen and J. R. Schrieffer, Phys. Rev. Lett. 78, 1771 (1997) [ARPES]; R. Nemetschek, M. Opel, C. Hoffmann, P. F. Müller, R. Hackl, H. Berger, L. Forró, A. Erb, and E. Walker, Phys. Rev. Lett. 78, 4837 (1997) [Raman]; G. V. M. Williams, J. L. Tallon, E. M. Haines, R. Michalak, and R. Dupree, Phys. Rev. Lett. 78, 721 (1997) [NMR]. 